\title{
Diffuse parenchymal lung disease as first clinical manifestation of GATA-2 deficiency in childhood
}

Tamara Svobodova ${ }^{1}$, Ester Mejstrikova ${ }^{2}$, Ulrich Salzer ${ }^{3}$, Martina Sukova ${ }^{2}$, Petr Hubacek ${ }^{2,4}$, Radoslav Matej, Martina Vasakova ${ }^{6}$, Ludmila Hornofova ${ }^{7}$, Marcela Dvorakova ${ }^{8}$, Eva Fronkova ${ }^{2}$, Felix Votava ${ }^{9}$, Tomas Freiberger ${ }^{10,11}$, Petr Pohunek', Jan Stary ${ }^{2}$ and Ales Janda $a^{3,12^{*}}$

\begin{abstract}
Background: GATA-2 transcription factor deficiency has recently been described in patients with a propensity towards myeloid malignancy associated with other highly variable phenotypic features: chronic leukocytopenias (dendritic cell-, monocyto-, granulocyto-, lymphocytopenia), increased susceptibility to infections, lymphatic vasculature abnormalities, and sensorineural deafness. Patients often suffer from opportunistic respiratory infections; chronic pulmonary changes have been found in advanced disease.
\end{abstract}

Case presentation: We present a case of a 17-year-old previously healthy Caucasian male who was admitted to the hospital with fever, malaise, headache, cough and dyspnea. A chest X-ray revealed bilateral interstitial infiltrates and pneumonia was diagnosed. Despite prompt clinical improvement under antibiotic therapy, interstitial changes remained stable. A high resolution computer tomography showed severe diffuse parenchymal lung disease, while the patient's pulmonary function tests were normal and he was asymptomatic. Lung tissue biopsy revealed chronic reparative and resorptive reaction with organizing vasculitis. At the time of the initial presentation to the hospital, serological signs of acute infection with Epstein-Barr virus (EBV) were present; EBV viremia with atypical serological response persisted during two-year follow up. No other infectious agents were found. Marked monocytopenia combined with B-cell lymphopenia led to a suspicion of GATA-2 deficiency. Diagnosis was confirmed by detection of the previously published heterozygous mutation in GATA2 (c.1081 C>T, p.R361C). The patient's brother and father were both carriers of the same genetic defect. The brother had no clinically relevant ailments despite leukocyte changes similar to the index patient. The father suffered from spondylarthritis, and apart from B-cell lymphopenia, no other changes within the leukocyte pool were seen.

Conclusion: We conclude that a diagnosis of GATA-2 deficiency should be considered in all patients with diffuse parenchymal lung disease presenting together with leukocytopenia, namely monocyto-, dendritic cell- and B-lymphopenia, irrespective of severity of the clinical phenotype. Genetic counseling and screening for GATA2 mutations within the patient's family should be provided as the phenotype is highly variable and carriers without apparent immunodeficiency are still in danger of developing myeloid malignancy. A prompt recognition of this rare condition helps to direct clinical treatment strategies and follow-up procedures.

Keywords: Primary immunodeficiency, GATA-2 deficiency, Diffuse parenchymal lung disease, EBV Viremia, Childhood

\footnotetext{
* Correspondence: ales.janda@uniklinik-freiburg.de

${ }^{3}$ Center for Chronic Immunodeficiency (CCI), University Medical Center and University of Freiburg, Freiburg im Breisgau, Germany

${ }^{12}$ Department of Pediatric Infectious Diseases and Rheumatology, Center of Pediatrics and Adolescent Medicine, University Medical Center and University of Freiburg, Mathildenstrasse 1, 79106 Freiburg im Breisgau, Germany

Full list of author information is available at the end of the article
} 


\section{Background}

Defects of transcription factor GATA-2 have recently been identified in a few overlapping phenotypes associated with myeloid malignancies: dendritic cell, monocyte, B- and NK-cell deficiency; MonoMAC syndrome (monocytopenia with Mycobacterium avium complex infections); Emberger syndrome (early onset primary lymphedema, multiple warts, sensorineural deafness, dysmorphism); and familial MDS/AML with no additional known phenotype. These syndromes share autosomal-dominant inheritance with variable manifestation of immunodeficiency [1-9]. The respiratory tract is frequently affected by viral, fungal or mycobacterial infections. Chronic lung tissue changes and pulmonary alveolar proteinosis (PAP), as well as pulmonary arterial hypertension, have been described in adult patients [1,7-9].

We present an adolescent male with GATA-2 deficiency and early manifestation of diffuse parenchymal lung disease
(DPLD) as well as an atypical course of Epstein-Barr virus (EBV) infection.

\section{Case presentation}

A 17-year-old Caucasian male presented to the hospital with acute fever, malaise, headache, cough and dyspnea. A bilateral pneumonia with signs of systemic inflammation corresponding to bacterial infection (C-reactive protein $210 \mathrm{mg} / \mathrm{l}$ ) was diagnosed and antibiotic treatment initiated. No causative microorganism was identified. Despite rapid clinical improvement, chest X-ray showed persistent interstitial changes (Figure 1A). A subsequent high-resolution computer tomography (HRCT) revealed marked lung damage suggestive of bronchiectasis with peribronchitis, fibrotisation, subpleural cystic remodeling (honey-combing) and emphysema (Figure 1B). Interestingly, pulmonary function tests showed normal vital capacity, total lung capacity as well as diffusing capacity (Table 1). Thus, we detected chronic lung

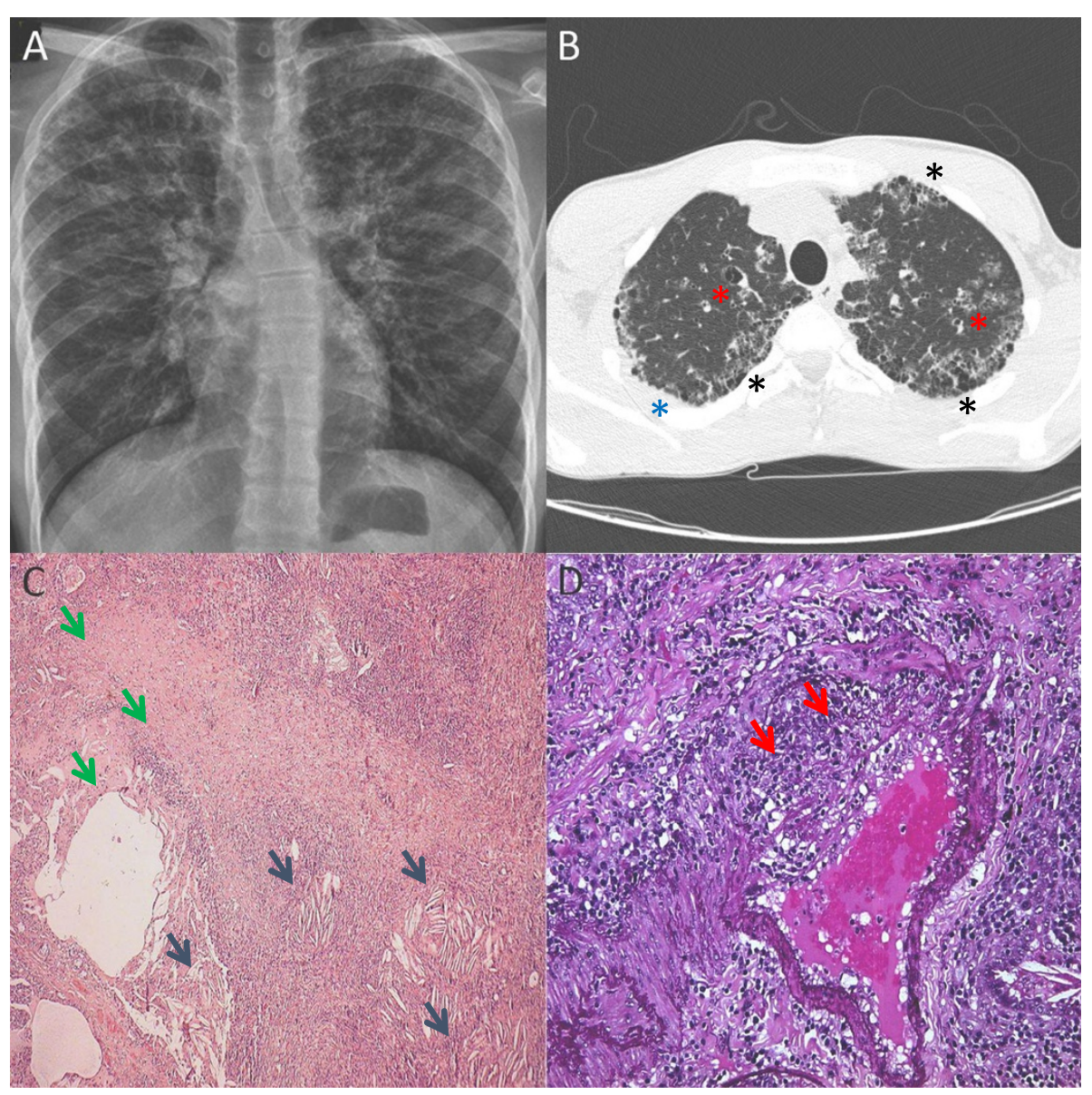

Figure 1 Pulmonary changes in the index patient. A: Diffuse bilateral linear and reticular opacities, compatible with interstitial pulmonary involvement (chest X-ray). B: Diffuse subpleural fibrotic changes - honeycomb (black asterisk), areas of subpleural consolidations (blue asterisk) and bronchectasis (red asterisk) in the upper lobes (high-resolution computer tomography scan). C: Chronic reparative and resorptive reaction: fibrosis and cystic rearrangement (green arrows) and cholesterol clefts (blue arrows) in the upper left lobe (hematoxylin and eosin tissue stain; original magnification 50x). D: Thickened arterial wall, destruction of the elastic layer, thrombosis showing organizing vasculitis (red arrows) in the upper left lobe (elastin tissue stain; original magnification 250x). 
Table 1 Results of pulmonary function tests

\begin{tabular}{lll}
\hline & Initial investigation & After 2-year follow-up \\
\hline FVC & 4.58 I (85\%) & 4.82 I (81\%) \\
FEV1 & 4.48 I/min (101\%) & $4.45 \mathrm{I} / \mathrm{min}(93 \%)$ \\
MEF50 & $7.32 \mathrm{I} / \mathrm{min}(140 \%)$ & $7.17 \mathrm{I} / \mathrm{min}(121 \%)$ \\
TLC & 6.14 I (92\%) & 6.09 I (78\%) \\
DLCO & $87 \%$ & $81 \%$
\end{tabular}

Legend: The first examination was performed two weeks after disease manifestation, there were more than 10 tests performed in the follow-up, all showing similar values. The numbers are related to normal values (parenthesis); the reference cohort has changed during the follow-up $(<18$ and $>18$ years of age). The shape of expiratory/inspiratory flow curve suggested intermittently mild periphery obstruction. Abbreviations: FVC Forced vital capacity, FEV1 Forced expiratory volume in 1 second, MEF Maximal mid-expiratory flow, TLC Total lung capacity, DLCO Diffusing lung capacity.

changes with no functional correlate during the first episode of pneumonia in a previously healthy boy. Further investigations to unfold the cause for the diffuse parenchymal lung disease were initiated.

A complete blood count showed leukocytopenia with marked monocytopenia (Table 2). Immunological assays detected B-cell lymphopenia with predominance of memory B cells. Despite the very low numbers of B cells, normal serum immunoglobulin levels of IgM and IgA and increased levels of IgG were present (23.6 g/l). Antibody response to routine vaccination was normal. No serum autoantibodies were found. Functional testing of granulocytes (respiratory burst test: analysis of the ability of granulocytes to release reactive oxygen species after in vitro stimulation) and of $\mathrm{T}$ cells (evaluation of proliferative response of $\mathrm{T}$ cells to various in vitro stimuli) excluded chronic granulomatous disease and T-cell proliferation defects.

Serology corresponded with primary EBV infection (Table 3). However, the EBV viral load in peripheral blood was low. Bronchoalveolar lavage (BAL) showed EBV presence in the bronchial fluid. Immunological analysis of the
BAL fluid showed lymphocytosis with predominance of CD8 ${ }^{\text {pos }}$ with increased HLA-DR expression (especially on $\mathrm{CD} 3{ }^{\text {pos }} 8^{\text {pos }}$ ); alveolar macrophages were present, CD1a ${ }^{\text {pos }}$ cells were not detected. No PAS (Periodic Acid-Schiff) positive material was evident in the alveolar macrophages (Table 4).

Neither bacterial, fungal, mycobacterial (including nontuberculous mycobacteria) nor viral (cytomegalovirus, human herpes virus 6, varicella zoster virus, human herpes virus, respiratory syntitial virus, influenza, adenovirus, enterovirus, coronavirus, parainfluenza, human rhinovirus, human metapneumovirus, bokavirus and papillomavirus tested) infection was revealed via culture, serology or molecular genetic testing in peripheral blood and bronchoalveolar fluid. Hence, the extensive microbiological analysis revealed only the presence of EBV in peripheral blood and lungs.

Histopathological investigation of the lung parenchyma was prompted. Thoracoscopic lung biopsy from a severely affected region of the right upper lobe showed fibrosis, cystic rearrangement and cholesterol clefts with signs of organizing pneumonia and vasculitis (Figure $1 \mathrm{C}, \mathrm{D}$ ). Inflammatory infiltration was predominantly lymphoplasmocytic with presence of activated macrophages. No changes compatible with pulmonary alveolar proteinosis or other alveolar filling disorder were seen. Despite an EBV presence (2300 copies/10.000 genomic equivalents, g.e.) in the lung tissue found with polymerase chain reaction, hybridization probes for EBV-encoded small RNA (EBER) were negative in the histology slides. Thus, no clear relationship between EBV and the histopathological parenchymal changes could be stated.

Given the severe affliction of the lung parenchyma with fibrotic remodeling, ongoing inflammation with activated CD8 ${ }^{\text {pos }} \mathrm{T}$ cells in the bronchoalveolar fluid and lack of clear evidence for an infectious cause, a treatment with an oral steroid was initiated to suppress further tissue destruction. A prophylactic antibiotic (azithromycin) was added and the

Table 2 Leukocyte and lymphocyte subsets

\begin{tabular}{|c|c|c|c|}
\hline & $\begin{array}{l}\text { Initial } \\
\text { investigation }\end{array}$ & $\begin{array}{l}\text { After 2-year } \\
\text { follow-up }\end{array}$ & Normal values \\
\hline & $\times 10^{\wedge} 9 / \mathrm{I}(\%)$ & $\times 10^{\wedge} 9 / \mathrm{l}(\%)$ & $\times 10^{\wedge} 9 / /(\%)$ \\
\hline Leukocytes & 2.8 & 5.4 & $4.0-10.0$ \\
\hline Monocytes & $0.014\left(0.5^{*}\right)$ & $0.028\left(0.5^{*}\right)$ & $0.12-1.0\left(3-10^{*}\right)$ \\
\hline Lymphocytes & $0.773\left(27.6^{*}\right)$ & $2.120\left(39.3^{*}\right)$ & $1.0-3.3\left(25-33^{*}\right)$ \\
\hline T cells (CD3+) & $0.659\left(85.3^{* *}\right)$ & $1.696\left(80.0^{* *}\right)$ & $2.0-6.5\left(55-88^{* *}\right)$ \\
\hline B cells (CD19+) & $0.015\left(1.9^{* *}\right)$ & $0.042\left(2.0^{* *}\right)$ & $0.4-3.3\left(11-45^{* *}\right)$ \\
\hline NK cells (CD56 + 16+) & $0.085\left(11.0^{* *}\right)$ & $0.382\left(18.0^{* *}\right)$ & $0.1-1.0\left(6-26^{* *}\right)$ \\
\hline CD4/8 index & 1.3 & 1.7 & $1-3$ \\
\hline
\end{tabular}

Legend: The initial leuko- and lymphopenia resolved; the profound monocyto- and B-cell lymphopenia persisted. The B cells were predominantly of memory phenotype. Myeloid as well as plasmacytoid dendritic cells were missing. In the parenthesis percentage of cells out of $(*)$ leukocytes and (**) lymphocytes, respectively, are shown. 
Table 3 Serologic and molecular genetic analysis of Epstein-Barr virus infection

\begin{tabular}{llll}
\hline & & Initial investigation & After 2-year follow-up \\
\hline Anti-VCA lgG & $\mathrm{U} / \mathrm{ml}$ & 63 & $>750$ \\
Anti-VCA lgM & $\mathrm{U} / \mathrm{ml}$ & $>160$ & 47 \\
Anti-EA-D lgG & $\mathrm{U} / \mathrm{ml}$ & 22 & $>150$ \\
Anti-EBNA 1 lgG & $\mathrm{U} / \mathrm{ml}$ & Negative & Negative \\
Viral load in PB & Copies/10000 g.e & 288 & 31 \\
Viral load in BAL & Copies/ml & 22000 & 6000 \\
\hline
\end{tabular}

Abbreviations: VCA viral capsid antigen, EA-D early antigen component, EBNA 1 Epstein-Barr virus nuclear antigen 1, PB peripheral blood, BAL bronchoalveolar fluid, g.e. genomic equivalent.

patient was closely monitored. Immunoglobulin levels normalized and signs of systemic inflammation regressed. After 6 months a stable finding was documented via HRCT and no clinical symptoms were present. The EBV viral load remained low in peripheral blood. Minimal presence of the virus was seen in repeated BAL. However, the serological signs of active EBV infection persisted and no EBNA antibodies were detected at the follow up. No lymphoproliferation was present and the patient remained asymptomatic. The condition was classified as persistent EBV viremia accompanied by an atypical serological response. Molecular genetic testing of $S H 2 D 1 A$ was carried out. A normal result excluded X-linked lymphoproliferative disease, the most common inborn cause of abnormal immunological reaction to EBV infection. Details on other possible genetic causes, not yet tested in our patient, are in the Discussion.

The patient has a history of occasional uncomplicated respiratory infections; At the age of 13 years he suffered from acute bronchitis, a chest X-ray was performed and retrospective analysis of the image showed some interstitial changes present already at that time. Monocytopenia was documented as early as at the age of 10 years.

The persistent profound monocytopenia and B- lymphocytopenia at follow up prompted GATA2 sequencing. The diagnosis of GATA-2 deficiency was confirmed by the finding of a known heterozygous pathogenic variation c.1081 C > T (p.R361C) [10]. Myeloid malignancy was excluded by morphological, flow cytometric and cytogenetic analysis of the bone marrow aspirate. Detailed immunophenotypic analysis of the bone marrow showed suppression of CD34 $4^{\text {pos }}$ and $\mathrm{CD} 117^{\text {pos }}$ precursors; impairment of B-cell lineage (only $1.4 \%$ B cells were present, out of those plasma cells

Table 4 Cytology analysis of bronchoalveolar fluid from the initial bronchoscopy investigation

\begin{tabular}{lll}
\hline Lymphocytes & $60.4 \%$ & of leukocytes \\
CD3 + DR+ & $35 \%$ & of CD3+ cells \\
CD4/8 ratio & 0.6 & \\
Neutrophile granulocytes & $6.8 \%$ & of leukocytes \\
Alveolar macrophages & $31.6 \%$ & of leukocytes \\
Plasmatic cells & $1.2 \%$ & of leukocytes \\
\hline
\end{tabular}

constituted $42 \%$ and mature $\mathrm{CD} 20^{\text {pos }} \mathrm{CD} 10^{\text {neg }}$ cells $37 \%$, the precursors $\mathrm{CD} 34^{\text {pos }} \mathrm{CD} 10^{\text {pos }}$ were scarce) and lower percentage of monocytes as well as their progenitors $\left(\mathrm{CD} 14^{\text {high }} \mathrm{CD} 45^{\text {pos }} \mathrm{SSC}{ }^{\text {med }}\right)$. Additional testing showed lack of myeloid and plasmacytoid dendritic cells.

The immunosuppressive treatment was stopped and the patient was further treated with prophylactic antibiotics and antimycotics. Vaccination against human papillomavirus (HPV) was performed as recommended [9]. He has been monitored closely, including regular checks of bone marrow aspirate for early detection of clonal myeloid proliferation. In case of myelodysplasia, transplantation of hematopoietic stem cells would be initiated. After two years of follow-up the patient did not develop any clinical symptoms. He was treated once for Pseudomonas aeruginosa found in the bronchoalveolar fluid detected in the second BAL analysis performed 6 months after the first one. Otherwise, there were no clinical signs of increased susceptibility to infection. Pulmonary function tests remained normal, no progression of the pulmonary parenchyma affliction have been detected so far (Tables 1, 2 and 3).

The same heterozygous mutation in GATA2 was found in the patient's 13-year-old brother and 45 year-old father, whereas his mother was healthy. The brother had been without any clinical symptoms so far, blood tests revealed leukocytopenia and marked monocytopenia. HRCT scan showed normal parenchyma, no EBV activity was documented. The father suffered from bilateral ankylosing spondylitis (HLA-B27 positive). Apart from low B-cell numbers (2.4\% CD $19^{\text {pos }}$ cells of lymphocytes, with prevailing memory phenotype: $70 \%$ CD27 $7^{\text {pos }}$ cells out of B cells; Norm $<47 \%$ ) no leukocyte count abnormalities were detected.

The lymphocyte changes in the three family members carrying the GATA2 mutation stimulated investigation of bone marrow output. Newly emerging $\mathrm{T}$ and $\mathrm{B}$ cells can be assessed via T-cell recombination circle (TREC) and kappadeleting element recombination circle (KREC) analysis in the peripheral blood $[11,12]$. As expected, both siblings had no detectable KREC copies in the peripheral blood, indicating severe impairment of B cell development. TREC analysis showed normal results. Bone marrow examination of the 
younger brother also showed complete negativity of KREC with normal TREC copies. KREC copies were absent in the peripheral blood of the father as well. The KREC/TREC copies were normal in the unaffected mother.

Interestingly, DNA obtained from the newborn Guthrie card of the younger brother was analyzed showing a normal amount of KREC/TREC copies. This indicates that the impairment in B lymphocyte development occurred postnatally.

\section{Discussion}

GATA-2 deficiency is a protean disease with a broad spectrum of symptoms. Most of the patients present with hematological abnormalities (cytopenias, early-onset myeloid malignancies) and an increased susceptibility to opportunistic infections [1-9]. In the recently published cohort of 57 patients treated at the National Institute of Health $(\mathrm{NIH}$, Bethesda, USA) [9] 70\% of the patients had severe viral infections, particularly infection with HPV (63\%) presenting with recalcitrant warts, condylomata, and/or dysplasia. Severe herpesvirus infections were present in $35 \%$ of patients: recurrent herpes stomatitis, esophagitis, genital infection, severe varicella in $11 \%$ of cases, and cytomegalovirus pneumonia or disseminated disease. Interestingly, in $11 \%$ of patients persistent EBV viremia similar to our patient was documented; in 2 patients EBV-positive skin tumors occurred. Infection with non-tuberculous mycobacteria was seen in 53\%, severe bacterial infection was observed in $49 \%$ and severe invasive fungal infection in $16 \%$ of the patients. Eighteen percent of patients showed no increased susceptibility to infection. Additionally, vascular/lymphatic defects (venous thrombosis, lymphedema), sensorineural hearing loss, miscarriages and hypothyroidism were found [1-9].

Pulmonary involvement in GATA-2 deficiency is frequent, involving infections and PAP [1,3,8,9], particularly in more advanced stages of the disease. In the NIH cohort, $79 \%$ and $63 \%$ of the patients had diffusion and ventilatory defects, respectively. PAP was found in $18 \%$ and pulmonary arterial hypertension in $9 \%$ of patients. Structural abnormalities included nodules, reticular and ground glass opacities, subpleural blebbing, "crazy paving", and paraseptal emphysema [9]. Similar picture could be seen in our patient. The surprisingly normal pulmonary function test results in our patient could possibly be explained by localized affliction of the pulmonary tissue. The infiltrated and fibrotic tissue decreased the elasticity of the lung parenchyma, however, there was still enough normal tissue that kept the static volumes and transfer factor normal (Table 1). Unfortunately, it is not possible to compare our findings with other pediatric patients as the data on pulmonary infliction in children are scarce. There were 24 children in the NIH cohort. Data on the pulmonary function tests were presented for only 6 of them (median age at testing 16 years, range $12-17$ years; median time from disease manifestation 2.5 years, range $0-16$ years). All those children suffered from myeloid malignancy, in four of them a chronic infection with herpesviruses or mycobacteria species was documented. Mild to severe diffusion defects were found in all tested patients, in two children a bronchial obstruction was seen. No information on structural lung changes in the affected children was provided [9].

The median age at initial presentation in the $\mathrm{NIH}$ cohort was 20 years but was highly variable (range 5 months 78 years). Of note, four individuals (7\%) had no apparent clinical manifestations as of the last follow-up (range 5-55 years). The proportion of patients without symptoms was $50 \%$ by age $20,25 \%$ by age 30 , and $16 \%$ by age 40 irrespective of the type of genetic change in GATA2. The phenotype varied within families significantly [9]. This fact strongly argues for a substantial impact of epigenetic, infectious and environmental factors on disease manifestation. Effects of germline or somatic mutations in other genes may play a role as well. This may explain the variability of symptoms in the three individuals carrying the same GATA2 mutation within our index family.

An intriguing issue is the etiology of the chronic diffuse parenchymal lung tissue changes in our patient in the absence of respiratory symptoms. The extensive investigations revealed only EBV presence in peripheral blood as well as in pulmonary tissue without specific tissue changes or clinically apparent EBV infection (e.g. mononucleosis-like symptoms, lymphoproliferation). As the patient presented with serological signs of acute EBV infection whereas the pulmonary changes were chronic, a decisive role of EBV in the pathogenesis of the pulmonary tissue changes in our patient was improbable. Possibly repeated mild infections in an environment of impaired regulation of the endothelial nitric oxide synthetase expression [13], defective phagocytosis and impaired GM-CSF signaling in pulmonary macrophages $[14,15]$ played a role in the pathogenesis of the chronic pulmonary inflammatory changes.

Poor control of EBV replication resulting in persistent EBV viremia irrespective of lung involvement is a known phenomenon in GATA-2 deficient patients [1,7-9]. It has been shown that the inability to confine viral infections in patients with GATA-2 deficiency correlates well with the extent of cytopenias, namely with the lack of DC-, NK- and $\mathrm{CD} 4^{\text {pos }}$ T-cells. Similarly, the defective antibody response at more advanced stages is associated with B cell lymphopenia $[8,9]$. However, with regards to the possible oligogenic etiology of immunodeficiency in GATA-2 deficiency, impact of other genes implicated in EBV control should be considered. We have excluded only the most common syndrome - Xlinked lymphoproliferative disease type 1 caused by a defect in an adapter protein SAP, involved in signalling of cell-cell interactions. Other molecules implicated in EBV control encompass for example: the ubiquitously expressed XIAP with 
both antiapoptotic function and multiple signalling pathway connections; the surface molecule CD27, important for intercellular communication; the NK cell activating receptor for antibody-dependent cell cytotoxicity (CD16), and minichromosome maintenance 4 (MCM4) crucial for NK-cell function; or IL-2-inducible T-cell kinase (ITK), coronin1A, serine-threonine kinase (STK)4 and magnesium transporter, MAGT1, indispensable for T-cell receptor signalling and Tcell homeostasis [16].

The search for a GATA-2 defect in our patient was prompted by the abnormalities in the leukocyte and lymphocyte counts. Another serum marker useful in diagnostics as well as in monitoring of the disease progression (correlating with cytopenia) is the stem cell growth Fmsrelated tyrosine kinase 3 ligand (Flt3 ligand) [8]. We have shown that the newborn screening using KREC/TREC analysis [17] cannot be used to screen for GATA-2 deficiency.

The prognosis of individuals with GATA2 mutations is difficult to establish due to high clinical variability, incomplete penetrance and lack of close phenotype-genotype correlation data $[8,9,18]$. Antibiotics (e.g. azithromycin) and HPV vaccination are the recommended prophylactic measures [9]. Use of steroids or other immunosuppressive therapy is not indicated and exclusion of immunodeficiency in DPLD prior to use is warranted. A large proportion of patients will develop myeloid malignancy later in life [1,7-9]. The only curative therapy is allogeneic hematopoietic stem cell transplantation. Two patients with GATA-2 deficiency with pulmonary involvement transplanted for advanced MDS were reported to have profited significantly from this procedure [7].

\section{Conclusion}

Diffuse parenchymal lung diseases are a heterogeneous group of disorders with an often insidious onset of symptoms [19]. The underlying immunodeficiency may not be apparent and an immunological and genetic work-up is required, in particular if abnormalities in peripheral leukocyte counts are revealed. As demonstrated in our patient, an aberrant immune response to common respiratory infections may result in diffuse lung disease with bronchial and bronchiolar damage, significant chronic changes of pulmonary parenchyma and fibrotic remodeling. The structural changes might be present prior to any severe infection.

Diffuse parenchymal lung disease may become the first manifestation of the GATA-2 deficiency. Early genetic diagnosis is critical to direct clinical management, prophylaxis, transplantation, and family screening.

\section{Consent}

Written informed consent was obtained from the patient and the family for publication of this Case report and any accompanying images. A copy of written consent is available for review by the Editor of this journal.

\section{Competing interests}

The authors declare that they have no competing interests.

\section{Authors' contributions}

TS identified the patient and drafted the manuscript. EM acquired and analysed the flow cytometry data and revised the manuscript. US and TF acquired and analysed the genetic data and revised the manuscript. MS analysed and interpreted the clinical and laboratory data and revised the manuscript. PH acquired and analysed the virology data and revised the manuscript. RM, MV and LH acquired and analysed data on pulmonary tissue pathology and revised the manuscript. MD acquired and analysed the radiology data and revised the manuscript. EF and FV analysed the KREC/TREC data and revised the manuscript. PP acquired and analysed the pulmonary function tests and revised the manuscript. JS provided supervision, analysed the clinical data and revised the manuscript. AJ is the corresponding author, he initiated the study and wrote the manuscript. All authors read and approved the manuscript.

\section{Acknowledgements}

The study was supported by MH CZ - DRO, University Hospital Motol, Prague, Czech Republic 00064203, NT13433-4/2012 and IGA NT/14534. Dr. Janda is a recipient of an unrestricted fellowship grant from the European Society for Immunodeficiencies (ESID) provided by Baxter. Dr. Fronkova received the L'Oreal for Women in Science Fellowship in 2013. We are grateful to Dr. Rachel Bortnick for editing services. The article processing charge was funded by the German Research Foundation (DFG) and the Albert Ludwigs University Freiburg in the funding programme Open Access Publishing.

\section{Author details}

'Department of Pediatrics, 2nd Faculty of Medicine, Charles University in Prague and University Hospital Motol, Prague, Czech Republic. ${ }^{2}$ Department of Pediatric Hematology and Oncology, 2nd Faculty of Medicine, Charles University in Prague and University Hospital Motol, Prague, Czech Republic. ${ }^{3}$ Center for Chronic Immunodeficiency (CCI), University Medical Center and University of Freiburg, Freiburg im Breisgau, Germany. ${ }^{4}$ Department of Medical Microbiology, 2nd Faculty of Medicine, Charles University in Prague and University Hospital Motol, Prague, Czech Republic. ${ }^{5}$ Department of Pathology and Molecular Medicine, Thomayer's University Hospital, Prague, Czech Republic. ${ }^{6}$ Department of Respiratory Medicine, Thomayer's University Hospital, Prague, Czech Republic. ${ }^{7}$ Department of Pathology and Molecular Medicine, 2nd Faculty of Medicine, Charles University in Prague and University Hospital Motol, Prague, Czech Republic. ${ }^{8}$ Department of Radiology, 2nd Faculty of Medicine, Charles University in Prague and University Hospital Motol, Prague, Czech Republic. 'Department of Pediatrics, 3rd Faculty of Medicine, Charles University in Prague and University Hospital Kralovske Vinohrady, Prague, Czech Republic. ${ }^{10}$ Molecular Genetics Lab, Centre for Cardiovascular Surgery and Transplantation, Brno, Czech Republic.

${ }^{11}$ Department of Clinical Immunology and Allergology, Medical Faculty, Masaryk University Brno, Brno, Czech Republic. ${ }^{12}$ Department of Pediatric Infectious Diseases and Rheumatology, Center of Pediatrics and Adolescent Medicine, University Medical Center and University of Freiburg,

Mathildenstrasse 1, 79106 Freiburg im Breisgau, Germany.

Received: 6 June 2014 Accepted: 27 January 2015

Published online: 10 February 2015

\section{References}

1. Vinh DC, Patel SY, Uzel G, Anderson VL, Freeman AF, Olivier KN, et al. Autosomal dominant and sporadic monocytopenia with susceptibility to mycobacteria, fungi, papillomaviruses, and myelodysplasia. Blood. 2010;115:1519-29.

2. Hsu AP, Sampaio EP, Khan J, Calvo KR, Lemieux JE, Patel SY, et al. Mutations in GATA2 are associated with the autosomal dominant and sporadic monocytopenia and mycobacterial infection (MonoMAC) syndrome. Blood. 2011;118:2653-5.

3. Bigley V, Haniffa M, Doulatov S, Wang XN, Dickinson R, McGovern N, et al. The human syndrome of dendritic cell, monocyte, B and NK lymphoid deficiency. J Exp Med. 2011;208:227-34.

4. Ostergaard P, Simpson MA, Connell FC, Steward CG, Brice G, Woollard WJ, et al. Mutations in GATA2 cause primary lymphedema associated with a 
predisposition to acute myeloid leukemia (Emberger syndrome). Nat Genet. 2011:43:929-31.

5. Pasquet M, Bellanné-Chantelot C, Tavitian S, Prade N, Beaupain B, LaRochelle $\mathrm{O}$, et al. High frequency of GATA2 mutations in patients with mild chronic neutropenia evolving to MonoMac syndrome, myelodysplasia, and acute myeloid leukemia. Blood. 2013;121:822-9.

6. Hsu AP, Johnson KD, Falcone EL, Sanalkumar R, Sanchez L, Hickstein DD, et al. GATA2 haploinsufficiency caused by mutations in a conserved intronic element leads to MonoMAC syndrome. Blood. 2013;121(19):3830-7. S1-7.

7. Cuellar-Rodriguez J, Gea-Banacloche J, Freeman AF, Hsu AP, Zerbe CS,

Calvo KR, et al. Successful allogeneic hematopoietic stem cell transplantation for GATA2 deficiency. Blood. 2011;118:3715-20.

8. Dickinson RE, Milne P, Jardine L, Zandi S, Swierczek SI, McGovern N, et at. The evolution of cellular deficiency in GATA2 mutation. Blood. 2014;123:863-74.

9. Spinner MA, Sanchez LA, Hsu AP, Shaw PA, Zerbe CS, Calvo KR, et al. GATA2 deficiency: a protean disorder of hematopoiesis, lymphatics, and immunity. Blood. 2014;123:809-21.

10. Hsu AP, Johnson KD, Falcone EL, Sanalkumar R, Sanchez L, Hickstein DD, et al. GATA2 haploinsufficiency caused by mutations in a conserved intronic element leads to MonoMAC syndrome. Blood. 2013;121:3830-7.

11. Weinberg K, Blazar BR, Wagner JE, Agura E, Hill BJ, Smogorzewska M, et al. Factors affecting thymic function after allogeneic hematopoietic stem cell transplantation. Blood. 2001;97:1458-66.

12. Van Zelm MC, Szczepanski T, van der Burg M, van Dongen JJM. Replication history of B lymphocytes reveals homeostatic proliferation and extensive antigen-induced B cell expansion. J Exp Med. 2007;204:645-55.

13. German Z, Chambliss KL, Pace MC, Arnet UA, Lowenstein CJ, Shaul PW. Molecular basis of cell-specific endothelial nitric-oxide synthase expression in airway epithelium. J Biol Chem. 2000;275:8183-9.

14. Bert AG, Johnson BV, Baxter EW, Cockerill PN. A modular enhancer is differentially regulated by GATA and NFAT elements that direct different tissue-specific patterns of nucleosome positioning and inducible chromatin remodeling. Mol Cell Biol. 2007;27:2870-85.

15. Lasbury ME, Tang X, Durant PJ, Lee CH. Effect of transcription factor GATA-2 on phagocytic activity of alveolar macrophages from Pneumocystis carinii-infected hosts. Infect Immun. 2003;71:4943-52

16. Rickinson AB, Long HM, Palendira U, Münz C, Hislop AD. Cellular immune controls over Epstein-Barr virus infection: new lessons from the clinic and the laboratory. Trends Immunol. 2014;35:159-69.

17. Borte S, von Döbeln U, Fasth A, Wang N, Janzi M, Winiarski J, et al. Neonatal screening for severe primary immunodeficiency diseases using highthroughput triplex real-time PCR. Blood. 2012;119:2552-5.

18. Mutsaers PG, van de Loosdrecht AA, Tawana K, Bodor C, Fitzgibbon J, Menko FH. Highly variable clinical manifestations in a large family with a novel GATA2 mutation. Leukemia. 2013;27:2247-8.

19. Clement A, Nathan N, Epaud R, Fauroux B, Corvol H. Interstitial lung diseases in children. Orphanet J Rare Dis. 2010;5:22.

\section{Submit your next manuscript to BioMed Central and take full advantage of:}

- Convenient online submission

- Thorough peer review

- No space constraints or color figure charges

- Immediate publication on acceptance

- Inclusion in PubMed, CAS, Scopus and Google Scholar

- Research which is freely available for redistribution 